\title{
The association of sleep quality with dry eye disease: the Osaka study
}

\author{
This article was published in the following Dove Press journal: \\ Clinical Ophthalmology \\ I June 2016 \\ Number of times this article has been viewed
}

\section{Motoko Kawashima' \\ Miki Uchino ${ }^{1,2}$ \\ Norihiko Yokoi ${ }^{3}$ \\ Yuichi Uchino ${ }^{1,2}$ \\ Murat Dogru' \\ Aoi Komuro 3 \\ Yukiko Sonomura ${ }^{3}$ \\ Hiroaki Kato 3 \\ Shigeru Kinoshita ${ }^{3}$ \\ Kazuo Tsubota'}

'Department of Ophthalmology, Keio University School of Medicine, Shinjuku-ku, ${ }^{2}$ Ryogoku Eye Clinic, Sumida-ku, Tokyo, ${ }^{3}$ Department of Ophthalmology, Kyoto Prefectural University of Medicine, Kyoto, Japan

Correspondence: Motoko Kawashima Department of Ophthalmology, Keio University School of Medicine, 35 Shinanomachi, Shinjuku-ku,

Tokyo 1608582, Japan

Tel +81353633866

Fax +8I 353633045

Email motoko-k@a3.keio.jp
Purpose: To investigate the association of dry eye disease with sleep quality.

Methods: In 2011, a cross-sectional survey was conducted among all the employees, mainly young and middle-aged Japanese office workers, who used visual display technology, at a company in Osaka, Japan ( $\mathrm{N}=672$; age range $=26-64$ years). The participants were classified according to the Japanese dry eye diagnosis criteria by dry eye examination results including the Schirmer test, fluorescein and lissamine green staining, tear film break-up time, and symptom questionnaire into three groups as follows: definite dry eye disease, probable dry eye disease, and no dry eye disease. To determine sleep quality, Japanese version of the Pittsburgh Sleep Quality Index (global score) was implemented. The global score (range $=0-21$ ) was calculated by summing seven sleep variable scores (scale, $0-3$ ); scores $\geq 5.5$ indicated poor sleep.

Results: The total mean global score was $5.1 \pm 2.3$ (completed $\mathrm{N}=383$ ); $45 \%$ of the dry eye disease participants reported having poor sleep quality, while $34 \%$ of the no dry eye disease participants did so, with a significant difference found in the global score $(P=0.002)$. Furthermore, a statistically significant association was observed between the global score and dry eye disease $(P=0.005)$.

Conclusion: Poor sleep quality is associated with dry eye disease, especially with dry eye symptoms.

Keywords: dry eye, sleep quality, symptom, questionnaire, Pittsburgh Sleep Quality Index, tear film break-up time, visual display terminals

\section{Introduction}

Dry eye disease (DED) is widely recognized as a multifactorial chronic disease that is highly prevalent in many countries, including Japan. DED has recently gained recognition as a public health problem. ${ }^{1,2}$ DED presents with various symptoms that significantly affect the quality of life and work ability, as well as the physical, social, and psychological well-being of an individual, by causing impaired visual function, psychological problems, or both. ${ }^{3-9}$

Ocular symptoms such as dryness, eyestrains, and blurry vision are commonly reported symptoms related to visual display terminal (VDT) users. ${ }^{10}$ VDT exposure has been increasing among office workers because of the rapid advancement of information technology. A recent large-scale epidemiologic study of office workers in Japan concluded that VDT work is considered one of the key risk factors for DED. ${ }^{1-13}$ These studies illustrate how significantly DED can impact a patient's functioning in the modern technological society.

On the other hand, sleep is essential to our physical and mental well-being, and the number of people with a sleep deficiency seems likely to increase. Sleep deficiencies are considered a public health problem. ${ }^{14,15}$ Furthermore, mental health maintenance 
has been increasingly recognized as critical with respect to the management of office workers, and several studies have shown that sleep quality is associated with the mental health of office workers. ${ }^{16}$ Recent studies also suggest that a sleep disturbance is associated with circadian rhythm disruption and even hypertension (HT) and metabolic syndrome. ${ }^{17-20}$ It has been reported that tear secretion and tear stability have a circadian rhythm. ${ }^{21,22} \mathrm{~A}$ study also suggested that the tear secretion system might be associated with the reninangiotensin system, ${ }^{23}$ as metabolic syndrome patients have lower tear secretion. ${ }^{24}$

Accordingly, we speculate that sleep quality is associated with DED. We conducted the present study to clarify the relation between poor sleep quality and DED. Thus, the aim of this study was to investigate the relationship between sleep quality and DED among VDT workers.

\section{Materials and methods}

In 2011, we conducted a cross-sectional survey of all the employees, mainly consisting of young and middle-aged Japanese office workers, who used VDT at a company in Osaka, Japan ( $\mathrm{N}=672$; age range $=26-64$ years). The detailed study protocols have been shown in previous reports, and the methods and grading scale used in this study have been previously published. ${ }^{13}$ All ophthalmic examinations were performed by seven ophthalmologists who were DED specialists. In order to unify assessment of objective evaluation, the DED specialists reconfirmed the DED scoring system before examination. We investigated the association of DED parameters (the Schirmer test-I values, tear film break-up time [BUT] test values, corneal and conjunctival staining scores, and symptoms) with physical activity level and sleep quality. Written informed consent was obtained from all participants. Candidates with a history of refractive surgery were excluded from the study. Contact lens wearers and those with meibomian gland dysfunction were included in this study. We defined the duration of VDT use (stratified, none to over 10 hours in 1-hour categories) including both office and beyond office hours and contact lens use (yes or no). Past/current history of certain common systemic diseases such as HT, diabetes mellitus, depression, and use of systemic medications was determined by asking the participants whether they had ever been told by their physicians that they had these conditions, or they had used any systemic medications, including antidepressants. The research followed the tenets of the Declaration of Helsinki, and this study design was approved by the Institutional Review Board of Ryogoku Eye Clinic in Tokyo, Japan.

\section{Diagnosis of DED}

On the basis of the dry eye examination results including the Schirmer test, fluorescein and lissamine green staining, BUT, and the symptom questionnaire, the participants were classified into the following three groups: definite DED, probable DED, and non-DED. A dry eye diagnosis was made according to the latest Japanese dry eye diagnostic criteria (2006) as follows: 1) presence of dry eye symptomatology; 2) presence of qualitative or quantitative disturbance of the tear film (Schirmer test $\leq 5 \mathrm{~mm}$ or BUT $\leq 5$ seconds); and 3 ) presence of keratoconjunctival epithelial damage (total score of fluorescein and lissamine green staining $\geq 3$ points). A diagnosis of definite DED required adherence to all the three criteria. Subjects fulfilling two of the three criteria were diagnosed with probable DED, and those fulfilling one or none of the three criteria were given the diagnosis of non-DED. ${ }^{13,25,26}$

\section{Ocular surface symptom questionnaire}

We used a 29-item questionnaire to evaluate the symptoms associated with dry eyes (Table S1). ${ }^{26,27}$ The questionnaire consisted of three categories: 12 questions on dry eye symptoms, nine on visual symptoms, and eight on environmental factors. Each question was answered with a frequency graded on a 5-point severity scale: 0 , always; 1 , often; 2 , sometimes; 3 , rarely; and 4, never. The total symptom score was calculated using the following formula: questionnaire score $=$ (sum of the scores from all the answered questions $\times 100$ )/(total number of questions answered $\times 4$ ). The validity and reliability of this questionnaire had been confirmed. ${ }^{26,27}$

\section{Sleep quality}

A Japanese version of the Pittsburgh Sleep Quality Index $(\mathrm{PSQI}-\mathrm{J})^{28}$ was used to measure the patients' recent sleep quality and sleep quantity. PSQI is an effective and useful instrument for measuring subjective sleep quality and sleep disturbances. ${ }^{29}$ The PSQI consists of 17 items, generated from seven components, including subjective sleep quality, sleep latency, sleep duration, habitual sleep efficiency, sleep disturbance, sleep medication usage, and daytime dysfunction. The score for each component ranged from 0 to 3 . The sum of the scores of these seven components provided a global PSQI score that ranged from 0 to 21. Generally, higher scores indicate poorer sleep. ${ }^{28,29}$ In PSQI-J, a cutoff score of $>5.5$ has a sensitivity of $80.0 \%-85.7 \%$ for various patient groups and a specificity of $86.6 \%$ for control subjects. ${ }^{30}$

\section{Statistical analysis}

Categorical variables were analyzed using the $\chi^{2}$ test, and continuous variables using Student's $t$-test. After adjusting 
for any possible confounders (sex, age, and VDT hours), analysis of variance was used to estimate the impact of DED on the PSQI score. Additional adjustment for HT and diabetes mellitus (which was not associated with PSQI score, data not shown) did not affect the findings and was not included in the final models. $P$-values $<0.05$ were considered significant. All statistical analyses were performed using Statistical Analysis Software, Version 9.2 (SAS Institute Inc., Cary, NC, USA).

\section{Results}

The survey response rate for completing the PSQI, the dry eye questionnaires, and the dry eye examinations was $57.0 \%(383 / 672)$. We did not observe any subjects who had been diagnosed with depression and anxiety in the present study. The respondents' characteristics are summarized in Table 1. In comparison with the non-DED group, there were more females in the DED group. Subjects in the DED group showed longer VDT use than those in non-DED group.

A comparison of the sleep quality between DED and non-DED groups is shown in Table 2. The total mean PSQI global score was $5.1 \pm 2.3$. In the DED group, $45 \%$ of the participants reported poor sleep quality, while $34 \%$ of those in the non-DED group reported the same, with a significant difference found in the global score between the DED (5.4 \pm 2.2$)$ and the non-DED groups (4.6 \pm 2.3 ; Student's $t$-test, $P=0.002$ ) (Table 2).

Next, we investigated the association between the DED parameters and the PSQI score. The abnormal Schirmer value group had a significantly higher rate of abnormal sleep quality than that of normal Schirmer value group $(P=0.021)$ (Table 3$)$. No significant differences were observed between the groups in PSQI scores, BUT, and ocular surface staining score. The dry eye symptom score was significantly higher in the abnormal sleep quality group $(28.2 \pm 15.3)$ than that in the normal sleep quality group $(22.1 \pm 12.2, P<0.001)$ (Figure 1$)$.

Furthermore, a significant association between the DED and the PSQI score $(P=0.005)$ (Table 4$)$ was found while analyzing models to evaluate the association between the PSQI score and the DED, after considering the possible influence of other factors known to be associated with DED (age, sex, and VDT hours).

\section{Discussion}

In this cross-sectional study, we found that sleep quality was associated with DED among the office workers, and $45 \%$ of the participants in the DED group reported poor sleep quality. The results of our study suggested that sleep disturbances seem to be an influencing factor of DED, especially with dry eye symptoms (Figure 1). PSQI-J score was significantly associated with $\operatorname{DED}(P=0.005)$. This study revealed that all patients with definitive DED had chronic discomfort present in eyes during wake and sleep.

Previous studies reported that a sleep disturbance because of sleep apnea syndrome, which is characterized by recurrent complete or partial upper airway obstructions during sleep, was associated with DED. ${ }^{31-33}$ Sjögren's syndrome is often

Table I Characteristics of the study respondents who completed the dry eye disease examination and the Japanese version of the Pittsburgh Sleep Quality Index questionnaire

\begin{tabular}{|c|c|c|c|c|c|c|}
\hline & \multicolumn{3}{|c|}{ DED (definite + probable) group } & \multirow[t]{2}{*}{ Non-DED } & \multirow[t]{2}{*}{ Total } & \multirow{2}{*}{$\begin{array}{l}P \text {-value } \\
\text { (DED vs non-DED }\end{array}$} \\
\hline & Definite & Probable & Subtotal & & & \\
\hline \multicolumn{7}{|l|}{ Total } \\
\hline $\mathrm{n}$ & 43 & 206 & 249 & 134 & 383 & \\
\hline \multicolumn{7}{|l|}{ Sex } \\
\hline Men, n (\%) & $21(48.8)$ & $128(62.1)$ & $149(59.8)$ & $103(76.9)$ & $252(65.8)$ & $0.001 *$ \\
\hline Women, n (\%) & $22(5 \mid .2)$ & $78(37.9)$ & $100(40.2)$ & $31(23.1)$ & 131 (34.2) & \\
\hline \multicolumn{7}{|l|}{ Age } \\
\hline Mean \pm SD & $40.8 \pm 7.1$ & $42.7 \pm 8.3$ & $42.4 \pm 8.1$ & $42.7 \pm 9.0$ & $42.5 \pm 8.4$ & $0.716 * *$ \\
\hline \multicolumn{7}{|l|}{ Age (category) } \\
\hline $20 s, n(\%)$ & $3(7.0)$ & $9(4.4)$ & $12(4.8)$ & $13(9.7)$ & $25(6.5)$ & $0.050 *$ \\
\hline 30 s, n (\%) & $16(37.2)$ & $65(31.6)$ & $81(32.5)$ & $31(23.1)$ & $112(29.2)$ & \\
\hline 40 s, n (\%) & $18(4 \mid .9)$ & $90(43.7)$ & $108(43.4)$ & $55(41.0)$ & $163(42.6)$ & \\
\hline $50 \mathrm{~s}, \mathrm{n}(\%)$ & $6(14.0)$ & $42(20.4)$ & $48(19.3)$ & $35(26.1)$ & $83(21.7)$ & \\
\hline \multicolumn{7}{|l|}{ VDT time } \\
\hline Mean \pm SD & $8.4 \pm 2.1$ & $8.1 \pm 2.3$ & $8.1 \pm 2.3$ & $7.6 \pm 2.1$ & $8.0 \pm 2.2$ & $0.020^{* *}$ \\
\hline \multicolumn{7}{|c|}{ VDT time (two categories) } \\
\hline$<8$ h, n (\%) & $31(72.1)$ & I 46 (70.9) & I77 (7I.I) & $113(84.3)$ & $290(75.7)$ & $0.006 *$ \\
\hline$\geq 8 \mathrm{~h}, \mathrm{n}(\%)$ & $12(27.9)$ & $60(29.1)$ & $72(28.9)$ & $21(15.7)$ & $93(24.3)$ & \\
\hline
\end{tabular}

Notes: $* \chi^{2}$ test; $* *$ Student's $t$-test.

Abbreviations: DED, dry eye disease; SD, standard deviation; VDT, visual display terminal; h, hours. 
Table 2 Sleep quality and dry eye disease

\begin{tabular}{|c|c|c|c|c|}
\hline & $\begin{array}{l}\text { DED (definite + } \\
\text { probable) }\end{array}$ & Non-DED & Total & $P$-value \\
\hline \multicolumn{5}{|c|}{ Sleep quality (PSQI-J) } \\
\hline \multicolumn{5}{|l|}{ Score } \\
\hline Mean \pm SD & $5.4 \pm 2.2$ & $4.6 \pm 2.3$ & $5.1 \pm 2.3$ & $0.002^{*}$ \\
\hline \multicolumn{5}{|l|}{ Category } \\
\hline Normal, n (\%) & $137(55.0)$ & $89(66.4)$ & 226 & $0.040^{* *}$ \\
\hline Abnormal, n (\%) & II $2(45.0)$ & $45(33.6)$ & 157 & \\
\hline
\end{tabular}

Notes: *Student's t-test; ** $\chi^{2}$ test.

Abbreviations: DED, dry eye disease; PSQI-J, Japanese version of the Pittsburgh Sleep Quality Index; SD, standard deviation.

complicated with severe DED, and Sjögren's syndrome patients tend to have sleep disturbances. It is considered to be related to the increased surface tension forces of the salivary and upper airway lining that are involved in the pathophysiology of obstructive sleep apnea. ${ }^{34-37}$ However, no significant correlations were found among the measures of sleep disruption, sleep apnea severity, and subjective sleep quality. ${ }^{37}$ The results obtained show that it is important to consider other possible explanations such as psychological factors because several studies have reported discrepancies between the subjective and objective evaluations of sleep $p^{37,38}$ (eg, the positive and negative affective states that are linked with physical health outcomes, as substantial evidence exists regarding the association of negative affective states with both sleep duration and quality). ${ }^{39-41}$

In this study, sleep disturbances were significantly associated with dry eye symptoms. One caveat of this study was that it utilized the PSQI, a self-reported questionnaire, to evaluate sleep quality. However, in this study, we revealed not only the dry eye symptoms, but also the diagnosis of DED. In addition, a low Schirmer value was significantly associated with sleep disturbance (Table 3). Consistent with this study results, poor sleep quality has been recently reported to be one of the risk factors for DED. ${ }^{42}$ In the People's Republic of China, a cross-sectional survey conducted among senior high school students demonstrated a significant association between sleep quality and DED. ${ }^{42}$ Meanwhile, mental health problems among office workers have been also increasingly acknowledged as a severe social problem, and sleep quality is considered to be involved in impairing mental health, ${ }^{16}$ as its association with sleep quality may be related to DED symptoms. For instance, depression, one of the major mental health conditions, is known to be associated with sleep disturbance, ${ }^{43}$ although no subjects reported having been diagnosed with depression or using antidepressants in the present study. Interestingly, depression has been reported to be associated with DED symptoms in people with normal or mildly reduced tear production. ${ }^{44}$ A study of the US Veterans Affairs population demonstrated that depression increases the risk of DED. ${ }^{33}$

Furthermore, the use of VDT including laptop computers, tablets, electronic readers, and smartphones is considered one of the strong risk factors of DED; in fact, it is well known that VDT use can induce various psychological and physiological adverse effects including various symptoms of DED. Consequently, DED is more prevalent in office workers who tend to have a high exposure to VDT, ${ }^{11}$ and has been reported to lead to the deterioration of work performance, ${ }^{9}$ which should be one of the crucial issues in business management. In addition, the use of electric lights at night, such as VDT use, leads to disrupted sleep for an increasing number of people. ${ }^{15}$ The daytime office environment also has an effect on sleep and may lead to sleep disturbance. ${ }^{45}$ In other words, less use of VDT may improve the symptoms of DED and sleep quality because less VDT exposure time reduces the risk of DED. ${ }^{11,12}$

These findings, however, should be viewed in light of several study limitations. Selection bias is one of the major limitations that should be considered because of the low response rate $(57.0 \%)$. We used a self-reported questionnaire to investigate sleep quality; therefore, future research should

Table 3 The Japanese version of the Pittsburgh Sleep Quality Index questionnaire score and dry eye objective parameters

\begin{tabular}{|c|c|c|c|c|c|}
\hline & \multirow[t]{2}{*}{$\mathbf{n}$} & \multicolumn{4}{|l|}{ PSQI-J score } \\
\hline & & $\begin{array}{l}<5.5 \text { (normal) } \\
226\end{array}$ & $\begin{array}{l}\geq 5.5 \text { (abnormal) } \\
\text { I } 57\end{array}$ & $\begin{array}{l}\text { Total } \\
383\end{array}$ & $\begin{array}{l}P \text {-value } \\
\left(\chi^{2} \text { test }\right)\end{array}$ \\
\hline \multirow[t]{2}{*}{ Schirmer value } & $\leq 5 \mathrm{~mm}$ (abnormal), $\mathrm{n}(\%)$ & $29(45.3)$ & & 64 & 0.021 \\
\hline & $>5$ mm (normal), n (\%) & $197(6 \mid .8)$ & $122(38.2)$ & 319 & \\
\hline \multirow[t]{2}{*}{ BUT } & $\leq 5 \mathrm{~s}$ (abnormal), n (\%) & $173(58.2)$ & & 297 & 0.662 \\
\hline & $>5$ s (normal), n (\%) & $53(61.6)$ & $33(38.4)$ & 86 & \\
\hline Ocular surface & $<3$ (normal), n (\%) & $187(58.6)$ & I $32(4 \mid .4)$ & 319 & 0.838 \\
\hline staining score & $\geq 3$ (abnormal), n (\%) & $39(60.9)$ & & 64 & \\
\hline
\end{tabular}

Abbreviations: BUT, tear film break-up time; PSQI-J, Japanese version of the Pittsburgh Sleep Quality Index; s, seconds. 


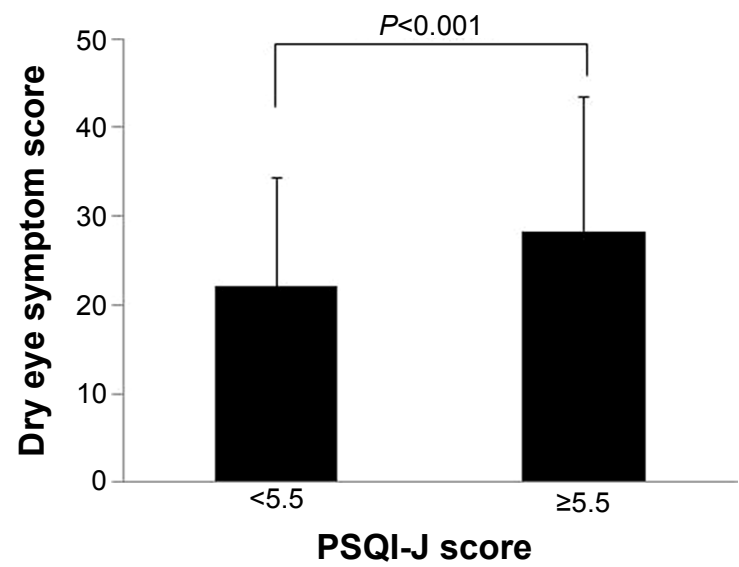

Figure I The association between sleep quality and dry eye symptom. Note: $P$-value was calculated with Student's $t$-test.

Abbreviation: PSQI-J, Japanese version of the Pittsburgh Sleep Quality Index.

be conducted implementing objective tools to evaluate sleep quality. Also, we would like to investigate subtypes of sleep disturbance and DED. In this study, we had no participants with sleep apnea, but such connection among DED, sleep disturbance, and sleep apnea should be investigated. Furthermore, as with all cross-sectional studies, a causal relationship remains unclear. In the future, given the results of this study, we should consider the impact of nighttime and daytime exposures to VDT separately on DEDs and examine those persons who have the same VDT exposure time. Also, the VDT work-related complaints such as headache and shoulder pain, in addition to DED, should be investigated about their effects on sleep as a prospective study.

\section{Conclusion}

In conclusion, although larger longitudinal or interventional trials are necessary, we found that sleep quality is associated with DED. A sleep disturbance seems to be an influencing factor on DED, especially on dry eye symptoms.

Table 4 Dry eye disease associated with the Japanese version of the Pittsburgh Sleep Quality Index questionnaire score and adjusted for other factors

\begin{tabular}{llllll}
\hline Source & df & SS & MS & $\boldsymbol{F}$ & P-value \\
\hline Dry eye & $\mathrm{I}$ & 40.027 & 40.027 & 7.96 & 0.005 \\
Sex & $\mathrm{I}$ & 2.700 & 2.700 & 0.54 & 0.464 \\
Age (four categories) & 3 & 29.549 & 9.850 & 1.96 & 0.120 \\
VDT working hours & $\mathrm{I}$ & $3.65 \mathrm{I}$ & $3.65 \mathrm{I}$ & 0.73 & 0.395 \\
(two categories) & & & & & \\
Error & 376 & $\mathrm{I}, 891.247$ & 5.030 & & \\
Total & 382 & $\mathrm{I}, 975.473$ & & & \\
\hline
\end{tabular}

Note: Adjusted for sex, age, and VDT working hours for each group.

Abbreviations: $d f$, degrees of freedom; F, F-value; MS, mean square; SS, sum of squares; VDT, visual display terminal.

\section{Acknowledgments}

We thank Dr Yuji Nishiwaki for his valuable advice.

This study was supported by a grant-in-aid from the Ministry of Education, Science, Sports, and Culture and Grant-in-Aid for Young Scientists (B), 2279192, 2010. Part of this study was sponsored by Santen Pharmaceutical Co., Ltd., Osaka. Provision of facilities and transport of equipment were supported by Santen Pharmaceutical Co., Ltd. The funders had no role in study design, data collection and analysis, decision to publish, or preparation of the manuscript.

We have the following interests. During the 36 months prior to submission, Dr Yokoi was a consultant for Kissei Co., Ltd. and Rohto Co., Ltd. Dr Kinoshita was a consultant for Santen Pharmaceutical Co., Ltd and Otsuka Pharmaceutical Co., Ltd. Dr Tsubota was a consultant for Santen Pharmaceutical Co., Ltd., AcuFocus, Inc, Bausch Lomb Surgical, Pfizer, and Thea. Dr Uchino Y, Dr Uchino M, Dr Komuro, Dr Kato, Dr Yokoi, Dr Sonomura, and Dr Kawashima received funding from a commercial source (Santen Pharmaceutical Co., Ltd). This does not alter our adherence to all the Clinical Ophthalmology policies on sharing data and materials.

\section{Disclosure}

The authors report no conflicts of interest in this work.

\section{References}

1. The epidemiology of dry eye disease: report of the Epidemiology Subcommittee of the International Dry Eye WorkShop. Ocul Surf. 2007; 5:93-107.

2. Brewitt H, Sistani F. Dry eye disease: the scale of the problem. Surv Ophthalmol. 2001;45(Suppl 2):S199-S202.

3. Pouyeh B, Viteri E, Feuer W, et al. Impact of ocular surface symptoms on quality of life in a United States Veterans Affairs population. Am J Ophthalmol. 2012;153:1061-1066.e3.

4. Mizuno Y, Yamada M, Miyake Y; Dry Eye Survey Group of the National Hospital Organization of Japan. Association between clinical diagnostic tests and health-related quality of life surveys in patients with dry eye syndrome. Jpn J Ophthalmol. 2010;54:259-265.

5. Li MY, Gong L. Progress of research on quality of life of dry eye patients Zhonghua Yan Ke Za Zhi. 2011;47:185-188.

6. Garcia-Catalan MR, Jerez-Olivera E, Benitez-Del-Castillo-Sanchez JM Dry eye and quality of life. Arch Soc Esp Oftalmol. 2009;84:451-458.

7. Friedman NJ. Impact of dry eye disease and treatment on quality of life. Curr Opin Ophthalmol. 2010;21:310-316.

8. Miljanovic B, Dana R, Sullivan DA, Schaumberg DA. Impact of dry eye syndrome on vision-related quality of life. Am J Ophthalmol. 2007;143: 409-415.

9. Yamada M, Mizuno Y, Shigeyasu C. Impact of dry eye on work productivity. Clinicoecon Outcomes Res. 2012;4:307-312.

10. Kawashima M, Uchino M, Kawazoe T, Kamiyashiki M, Sano K, Tsubota K. A field test of a web-based screening for dry eye disease to enhance awareness of eye problems among general internet users: a latent strategy to promote health. J Med Internet Res. 2013;27:15:e209.

11. Uchino M, Schaumberg DA, Dogru M, et al. Prevalence of dry eye disease among Japanese visual display terminal users. Ophthalmology. 2008;115:1982-1988. 
12. Uchino M, Nishiwaki Y, Michikawa T, et al. Prevalence and risk factors of dry eye disease in Japan: Koumi study. Ophthalmology. 2011;118: 2361-2367.

13. Uchino M, Yokoi N, Uchino Y, et al. Prevalence of dry eye disease and its risk factors in visual display terminal users: the Osaka study. Am J Ophthalmol. 2013;156:759-766.

14. Scully T. Sleep. Nature. 2013;497:S1-S3.

15. Czeisler CA. Perspective: casting light on sleep deficiency. Nature. 2013;497:S13.

16. Doi Y, Minowa M, Tango T. Impact and correlates of poor sleep quality in Japanese white-collar employees. Sleep. 2003;26:467-471.

17. Gangwisch JE. Epidemiological evidence for the links between sleep, circadian rhythms and metabolism. Obes Rev. 2009;10(Suppl 2): 37-45.

18. Wolk R, Somers VK. Sleep and the metabolic syndrome. Exp Physiol. 2007;92:67-78.

19. Plante GE. Sleep and vascular disorders. Metabolism. 2006;55(Supp12): S45-S49.

20. Esquirol Y, Perret B, Ruidavets JB, et al. Shift work and cardiovascular risk factors: new knowledge from the past decade. Arch Cardiovasc Dis. 2001;104:636-668.

21. Walker PM, Lane KJ, Ousler GW 3rd, Abelson MB. Diurnal variation of visual function and the signs and symptoms of dry eye. Cornea. 2010; 29:607-612.

22. Piccione G, Giannetto C, Fazio F, Giudice E. Daily rhythm of tear production in normal horse. Vet Ophthalmol. 2008;11(Supp1 1):57-60.

23. Yaguchi S, Ogawa Y, Shimmura S, et al. Angiotensin II type 1 receptor antagonist attenuates lacrimal gland, lung, and liver fibrosis in a murine model of chronic graft-versus-host disease. PLoS One. 2013;8:e64724.

24. Kawashima M, Uchino M, Yokoi N, et al. Decreased tear volume in patients with metabolic syndrome: the Osaka study. Br J Ophthalmol. 2014;98:418-420.

25. Shimazaki J, Tsubota K, Kinoshita S, Ohashi Y. [Definition and diagnosis of dry eye 2006.] Atarashii Ganka. 2007;24:181-184. Japanese.

26. Uchino Y, Uchino M, Dogru M, Ward S, Yokoi N, Tsubota K. Changes in dry eye diagnostic status following implementation of revised Japanese dry eye diagnostic criteria. Jpn J Ophthalmol. 2012;56:8-13.

27. Kojima T, Ibrahim OM, Wakamatsu T, et al. The impact of contact lens wear and visual display terminal work on ocular surface and tear functions in office workers. Am J Ophthalmol. 2011;152:933-940.

28. Doi Y, Minowa M, Okawa M. [Development of the Japanese version of the Pittsburgh Sleep Quality Index.] Jpn J Psychiatr Treat. 1998;13: 755-763. Japanese.

29. Buysse DJ, Reynolds CF 3rd, Monk TH, Berman SR, Kupfer DJ. The Pittsburgh Sleep Quality Index: a new instrument for psychiatric practice and research. Psychiatry Res. 1989;28:193-213.

30. Doi Y, Minowa M, Okawa M, Uchiyama M. Prevalence of sleep disturbance and hypnotic medication use in relation to sociodemographic factors in the general Japanese adult population. J Epidemiol. 2000; 10:79-86.
31. Karger RA, White WA, Park WC, et al. Prevalence of floppy eyelid syndrome in obstructive sleep apnea-hypopnea syndrome. Ophthalmol. 2006;113:1669-1674

32. Liu DT, Di Pascuale MA, Sawai J, Gao YY, Tseng SC. Tear film dynamics in floppy eyelid syndrome. Invest Ophthalmol Vis Sci. 2005;46: 1188-1194.

33. Galor A, Feuer W, Lee DJ, et al. Prevalence and risk factors of dry eye syndrome in a United States Veterans Affairs population. Am J Ophthalmol. 2011;152:377-384.

34. Hilditch CJ, McEvoy RD, George KE, et al. Upper airway surface tension but not upper airway collapsibility is elevated in primary Sjogren's syndrome. Sleep. 2008;31:367-374.

35. Kirkness JP, Madronio M, Stavrinou R, Wheatley JR, Amis TC. Surface tension of upper airway mucosal lining liquid in obstructive sleep apnea/ hypopnea syndrome. Sleep. 2005;28:457-463.

36. Jokic R, Klimaszewski A, Mink J, Fitzpatrick MF. Surface tension forces in sleep apnea: the role of a soft tissue lubricant: a randomized doubleblind, placebo-controlled trial. Am J Respir Crit Care Med. 1998;157: 1522-1525.

37. Usmani ZA, Hlavac M, Rischmueller M, et al. Sleep disordered breathing in patients with primary Sjogren's syndrome: a group controlled study. Sleep Med. 2012;13:1066-1070.

38. Jackowska M, Dockray S, Hendrickx H, Steptoe A. Psychosocial factors and sleep efficiency: discrepancies between subjective and objective evaluations of sleep. Psychosom Med. 2011;73:810-816.

39. Foley D, Ancoli-Israel S, Britz P, Walsh J. Sleep disturbances and chronic disease in older adults: results of the 2003 National Sleep Foundation Sleep in America Survey. J Psychosom Res. 2004;56:497-502.

40. Bliwise DL, King AC, Harris RB, Haskell WL. Prevalence of self-reported poor sleep in a healthy population aged 50-65. Soc Sci Med. 1992;34: 49-55.

41. Benca RM, Obermeyer WH, Thisted RA, Gillin JC. Sleep and psychiatric disorders. A meta-analysis. Arch Gen Psychiatry. 1992;49:651-670.

42. Zhang Y, Chen H, Wu X. Prevalence and risk factors associated with dry eye syndrome among senior high school students in a county of Shandong Province, China. Ophthalmic Epidemiol. 2012;19:226-230.

43. Cabello M, Mellor-Marsa B, Sabariego C, Cieza A, Bickenbach J, Ayuso-Mateos JL. Psychosocial features of depression: a systematic literature review. J Affect Disord. 2012;141:22-33.

44. Kim KW, Han SB, Han ER, et al. Association between depression and dry eye disease in an elderly population. Invest Ophthalmol Vis Sci. 2011; 52:7954-7958.

45. Kozaki T, Miura N, Takahashi M, Yasukouchi A. Effect of reduced illumination on insomnia in office workers. J Occup Health. 2012;54: 331-335. 


\section{Supplementary material}

Table SI Newly designed Dry Eye Symptom Questionnaire

Three-part questionnaire

A Symptom frequency and severity scores for dryness

I) Do you feel dryness in your eyes?

2) Are your eyes uncomfortable?

3) Do you experience a foreign body sensation in your eyes?

4) Do you have any pain in your eyes?

5) Do you feel any eyestrain?

6) Do you have any discomfort in your eyes?

7) Are your eyes bloodshot?

8) Do you have any itching in your eyes?

9) Do you have any difficulty opening your eyes?

10) Do you have any irritation in your eyes?

II) Do your eyes feel heavy?

12) If you have any other symptoms, please write it in the parentheses beneath and circle the frequency

Total (I-12): ( ) - A

B Symptom frequency severity scores for visual disturbance

13) Do you have hazy vision?

14) Do you have any glare?

15) While reading, do your dry eye symptoms worsen, and it becomes hard to continue reading?

16) While driving, do your dry eye symptoms worsen, and it becomes difficult to drive?

17) While operating the computer, do your dry eye symptoms worsen, and it becomes difficult to continue?

18) While watching TV or movies, do your dry eye symptoms worsen, and it becomes difficult to watch?

19) Do you have any after-images?

20) Do you think that blinking affects your sight?

21) Can you keep your eyes open without blinking for 10 seconds or more?

Total (|3-2|): ( ) - B

C Symptom frequency severity scores for environmental and lifestyle factors: Do your dry eye symptoms worsen?

22) When the wind is strong?

23) When it is dry in winter or summer?

24) When the air conditioning is on?

25) While flying?

26) When you feel stressed in daily life?

27) After alcohol consumption?

28) When you smoke or are exposed to someone smoking next to you?

29) When you are wearing contact lenses?

Total (22-29): ( ) - C

Notes: Of the 29 questions, I through 29, except for 2I, were classified into the following five stages: 4, always; 3, often; 2, occasionally; I, rarely; and 0, never. Question 2 was classified into the following five stages: 4 , not possible; 3 , with difficulty; 2 , moderately possible; I, probable; and 0 , not a problem.

Clinical Ophthalmology

\section{Publish your work in this journal}

Clinical Ophthalmology is an international, peer-reviewed journal covering all subspecialties within ophthalmology. Key topics include: Optometry; Visual science; Pharmacology and drug therapy in eye diseases; Basic Sciences; Primary and Secondary eye care; Patient Safety and Quality of Care Improvements. This journal is indexed on

PubMed Central and CAS, and is the official journal of The Society of Clinical Ophthalmology (SCO). The manuscript management system is completely online and includes a very quick and fair peer-review system, which is all easy to use. Visit http://www.dovepress.com/ testimonials.php to read real quotes from published authors.

\section{Dovepress}

